\title{
Approach to a Guideline for Epigenetic Therapy in the High Risk Cancer Setting
}

\author{
Saeed Taheri* \\ The New Lahijan Scientific Foundation, Iran
}

*Corresponding author: Taheri S. The New Lahijan Scientific Foundation; Kordestan Str; PO Box: 14376-45685, Tehran, Iran; Email: taherimd@gmail.com

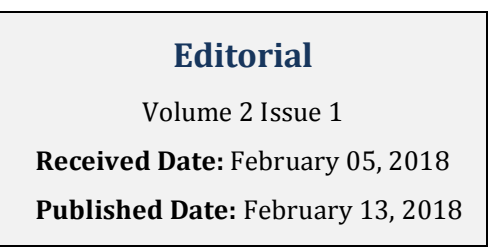

\section{Monotherapy with DNA Methyltransferase Inhibitors (DNMTi)}

Abbreviations: DNMTI: DNA Methyl Transferase CTCL: Cutaneous T-Cell Lymphoma; PTCL: Peripheral TCell Lymphoma; SCT: Stem Cell Transplant; AML: Acute Myeloid Leukemia; CMML: Chronic Myelomonocyticleukaemia; NHL: Non-Hodgkin Lymphoma; HL: Hodgkin's Lymphoma; NSCLC: NonSmall Cell Lung Cancer; TET: ten-eleven translocation

\section{Introduction}

The term 'epigenetics' had been originally described by Waddington in 1939; and after substantial changes in the definition, it has been finally defined as "the study of "heritable changes in gene expression, capable of altering cellular phenotype and function independent of alterations in DNA sequence", which has now been a focus of intensive research as a promising ambition for the management of malignant disorders [1]. There has been a large number of clinical trials investigating effects of therapeutic agents targeting epigenome of cancer patients, though the majority of them are early phase studies and there is scarcity of powerful data coming from randomized clinical trials with large patient populations in this setting. However, despite their less strong data coming from early phase studies, when the study is about patients of hard to manage categories, including older patients or those who have already shown refraction to the conventional therapy or relapsed after the primary treatment course, any promising result even if coming from less robust methodologies could be considered valuable or even inevitable. To address this issue, this editorial aims to provide an overview on the promising evidence on the positive effects of epigenetic treatments in the higher risk cancer patients, as defined above, and also to offer an abstracted approach to a guideline for targeted epigenetic therapy in this population, based on the current available data in the literature.
Although cancer tissues commonly experience global hypomethylation of their genome, focal hypermethylations occurring in the promoter CpG islands leads to silencing of several critical TSGs. This factor alongside the silencing of oncogenes through methylation of their genes bodies (and not promoters) as well as, silencing the second allele of a TSG gene in a Knudson's two-hit models makes DNMTi agents valuable in the context of cancer therapy [2]. DNMTi monotherapy has usually been investigated in the hematological malignancies, but it has been of very limited -if any- use in the non-hematological cancers.FDA has already approved azacitidine and decitabine for using in a number of hematological malignancies; and while a systematic review suggests more promising therapeutic efficacy for azacitidine versus decitabine, there is on the other hand evidence suggestive of decitabine efficacy in azacitidine-resistant MDS [3-4]. The most rigorous evidence about efficacy of DNMTi monotherapy in cancer patients comes from two pivotal clinical trials, AZA-MDS-001 and AZA-AML-001, whose data served the main evidence for the approval of these drugs for use in hematological diseases. Some of the most important clinical studies with positive responses reported for DNMTi therapy in cancer highrisk populations have been presented in Table 1.

\section{Monotherapy with Histone deacetylase inhibitors (HDACI)}

Histone dacetylases are a group of enzymes that eliminate acetyl groups from histones and can regulate expression of tumor suppressor genes, and HDACi agents inhibit their activity. To date, three HDACi agents have been approved for cancer therapy by the FDA that includes Vorinostat (SAHA, Zolina), for use in patients with cutaneous T-Cell Lymphoma (CTCL), a rare type of 
non-Hodgkin's lymphoma of the skin; romidepsin (Istodax, FK228, FR901228, depsipeptide), for the treatment of T-cell lymphoma, and belinostat (Beleodaq, PXD-101), for the treatment of patients with relapsed or refractory peripheral T-cell lymphoma (PTCL) [5].Although HDACi monotherapy in hematological and lymphoproliferative malignancies had been associated with promising results, the outcome of clinical trials in patients with solid tumors, except for individual reports with some modest effects, had been disappointing. For example, as a study with a most encouraging result, a phase II trial of patients with recurrent glioblastomamultiforme, vorinostat mono therapy resulted in progression free survival in only 9 out of 52 patients [6].Two invaluable review articles have recently published studying effects of DNMTi agents on solid tumors which can be used for more information [7-8]. Some of the most promising results coming from clinical trials on the impact of HDACi therapy in the hard to manage cancer patients is included in Table1.

\section{Clinical Evidence for DNMTi Plus HDACi Therapy Efficacy}

Although neither DNMTi nor HDACi agents represented any encouraging outcomes in the management of solid tumors when administered as single agent, dual therapy of solid tumors employing agents from these two groups had satisfactory results. On the other hand, results of this combination therapy in hematological cancers were more disappointing [9]. An explanation for this observation had been provided by Prebet et al., which speculated on the cell-cycle inhibition potency of some HDAC inhibitors and its potential inhibitory impact on the DNMTi incorporation into the DNA, when administered concomitantly, leading to poorer outcomes than monotherapy with DNMTi [10]. Some of the most successful concomitant employment of DNMTi and HDACi in the high-risk cancer patients is represented in Table 1.

\section{Combination of epigenetic agents with chemotherapy}

Chemotherapy is the backbone of patient management in most malignant disorders; nevertheless, its application is not always pertinent due to either the magnitude of its efficacy or associated side effects, in different cancer subpopulations. The epigenetic agents, despite their limitations of use, including own side effects as well as efficacy power, have been shown to be safe and efficacious in specific subpopulations of cancer patients, when administered concomitant or sometimes before the conventional chemotherapy. Selected studies representing some of the most promising evidence from the literature in this regard could be found in Table 1.

\section{Cytogenetic and Epigenetic Predictors}

Considering the principle topic of the current study, maybe the most important factor that may predict response to therapy in cancer patients is epigenomic status of their malignant lesions. In a comprehensive systematic review on microRNAs and their association to chemotherapy efficacy in gastric cancer cell lines, this author found an interesting similarity in the target genes of microRNAs that most greatly affect the same chemotherapy agent, suggesting that dysregulation of particular genes may predict resistance or sensitivity to specific chemotherapy agents [11]. So, it would not be surprising if particular cytogenetic or epigenetic signature of a cancer lesion well predict efficacy of a specific epigenetic-and/or chemo-therapy agent in those patients. For example, azacitidine is suggested to improve response rates in AML patients with ten-eleven translocation 2 (TET2) mutations compared with its wild type, while patients withTP53 mutations represent poorer overall survival in response to azacitidine therapy than those without the mutation [reviewed by ref 12]. Based on this fact, some prognostic scoring systems have been developed to predict cancer therapy results. A review article by Treppendahl et al. has listed molecular predictors for response to epigenetic therapy in cancer patients, and those fit this paper can be found in Table 1 [13].

\section{Conclusion}

\section{Approach to a Guideline development}

In order to develop a guideline for targeted use of epigenetic therapy in cancer patients, we would need robust evidence coming from studies employing rigorous methodology and enough sample size; however studies investigating efficacy of epigenetic therapy in cancer patients mainly are of early phase and include limited number of patients. On the other hand, there are studies investigating this effect in patients of higher risk settings, for whom there are few or no therapeutic choices are available. In these cases, at least, promising, data coming from even less powerful methodologies might worth to try in the clinical practice, until more vigorous data gets into enters the literature. In Table 1, a list of epigenetic therapies which had been associated with clinical response to epigenetic treatment in high-risk cancer patients is provided. The main approach in this paper was to exclude studies with very limited number of participants unless those with the most important, powerful results, or most distinctive cancer subgroups. Studies with the highly robust methodologies have been mentioned in bold 
font. Further A phase III studies in these cancer subpopulations empower us to prepare a more reliable guideline to manage these patients more steadily.

\begin{tabular}{|c|c|c|c|c|c|}
\hline Cancer type & Subpopulation & $\mathbf{n}$ & Treatment & Limitation/power & $\begin{array}{c}\text { Ref. } \\
\text { (clinicaltrials.gov) }\end{array}$ \\
\hline \multirow{10}{*}{ AML/MDS } & Elderly & 488 & $\begin{array}{c}\text { Azacitidine } \\
\text { [Approved by FDA, } \\
\text { EMA] }\end{array}$ & $\begin{array}{c}\text { Survival benefit/Phase } \\
\text { III trial }\end{array}$ & NCT01074047 \\
\hline & $\begin{array}{c}\text { Elderly with high miR-29 } \\
\text { level }\end{array}$ & 53 & decitabine & Clinical response & NCT00492401 \\
\hline & $\begin{array}{c}\text { Elderly with poor/interm. } \\
\text { cytogenetics }\end{array}$ & 485 & $\begin{array}{c}\text { Decitabine } \\
\text { [Approved by FDA, } \\
\text { EMA] }\end{array}$ & $\begin{array}{c}\text { Phase III trial/clinical } \\
\text { response/NSS survival } \\
\text { benefit }\end{array}$ & NCT00260832 \\
\hline & $\begin{array}{c}\text { Elderly + multiple } \\
\text { comorbidities }\end{array}$ & 227 & Azacitidine(vs. IC) & $\begin{array}{c}\text { Less side effects/similar } \\
\text { survival }\end{array}$ & $\begin{array}{c}\text { doi: } \begin{array}{c}10.1186 / 1756- \\
8722-6-29\end{array} \\
\end{array}$ \\
\hline & Elderly & 50 & \begin{tabular}{|c|}
$\begin{array}{c}\text { Azacitidine }+ \\
\text { pracinostat }\end{array}$ \\
\end{tabular} & \begin{tabular}{|c|} 
Clinical response/Nd. \\
RND
\end{tabular} & NCT01912274 \\
\hline & Elderly non-fit AML & 204 & \begin{tabular}{|c|} 
Decitabine + (ATRA vs. \\
VPA)
\end{tabular} & $\begin{array}{c}\text { Survival } \\
\text { benefit/randomized trial }\end{array}$ & NCT00867672 \\
\hline & Poor cytogenetics & 358 & \begin{tabular}{|c|}
$\begin{array}{c}\text { Azacitidine (vs. } \\
\text { LDAC/CCR) }\end{array}$ \\
\end{tabular} & \begin{tabular}{|c|} 
Phase III/Much better \\
survival
\end{tabular} & NCT00071799 \\
\hline & Relapsed/refractory & 66 & $\begin{array}{l}\text { Mocetinostat + } \\
\text { azacitidine }\end{array}$ & $\begin{array}{c}\begin{array}{c}\text { Phase II study; } 2 / 3^{\text {rd }} \\
\text { of patients achieved } \\
\text { CR/nd. RND }\end{array} \\
\end{array}$ & NCT00324220 \\
\hline & Relapsed/refractory & 36 & Decitabine + ATRA & Good survival/nd. RND & $\begin{array}{c}\text { doi: } 10.1007 / \mathrm{s} 00277 \\
016-2681-3 \\
\end{array}$ \\
\hline & $\begin{array}{l}\text { Relapsed/refractory/Respon } \\
\text { se only in CMML subgroup }\end{array}$ & 260 & $\begin{array}{l}\text { Azacitidine }+ \\
\text { lenalidomide }\end{array}$ & \begin{tabular}{|c|} 
Clinical \\
response/randomized \\
trial
\end{tabular} & NCT01617226 \\
\hline \multirow{7}{*}{ Lymphoma } & Relapsed/refractory NHL & 35 & Vorinostat & $\begin{array}{c}9 \% \text { ORR, } 20 \text { mo PFS/nd } \\
\text { RND }\end{array}$ & NCT00253630 \\
\hline & Relapsed/refractory HL & 37 & Resminostat & \begin{tabular}{|c|}
$\sim 50 \%$ clinical \\
response/Nd. RND \\
\end{tabular} & NCT01037478 \\
\hline & Relapsed/refractory PTCL & 74 & $\begin{array}{c}\text { Romidepsin } \\
\text { [Approved by FDA] }\end{array}$ & $\begin{array}{c}\sim 25 \% \text { clinical } \\
\text { response/nd. RND }\end{array}$ & NCT00091559 \\
\hline & Relapsed/refractory PTCL & 47 & Romidepsin & $\begin{array}{l}\text { 38\% response rate/nd. } \\
\text { RND }\end{array}$ & NCT00007345 \\
\hline & $\begin{array}{c}\text { Relapsed/refractory HL after } \\
\text { autologous SCT }\end{array}$ & 129 & Panobinostat & $\begin{array}{c}\text { Tumor reduction in } \\
74 \% ; 1 \text { yr survival in } \\
78 \% / \text { nd. RND } \\
\end{array}$ & NCT00742027 \\
\hline & \begin{tabular}{|c|} 
Progressive/relapsed \\
peripheral T cell lymphoma
\end{tabular} & 131 & Romidepsin & $\sim 25 \%$ Clinical response & NCT00426764 \\
\hline & $\begin{array}{c}\text { Relapsed/refractory } \\
\text { lymphoma }\end{array}$ & 29 & $\begin{array}{c}\text { Vorinostat + rituximab } \\
+ \text { ifosfamide }+ \\
\text { carboplatin }+ \\
\text { etoposide }\end{array}$ & $\begin{array}{c}\sim 2 / 3^{\text {rd }} \text { of patients } \\
\text { clinically responded } / \text { nd. } \\
\text { RND }\end{array}$ & NCT00601718 \\
\hline \multirow{3}{*}{ Ovary } & $\begin{array}{c}\text { Platinum- } \\
\text { resistant/refractory }\end{array}$ & 30 & \begin{tabular}{c|}
$\begin{array}{c}\text { Azacitidine }+ \\
\text { carboplatin }\end{array}$ \\
\end{tabular} & \multicolumn{2}{|c|}{ PRR:22\%, median OS: 23doi: 10.1002/cncr.25 } \\
\hline & $\begin{array}{l}\text { Platinum- } \\
\text { resistant/refractory }\end{array}$ & 28 & $\begin{array}{l}\text { Decitabine + } \\
\text { carboplatin }\end{array}$ & \begin{tabular}{|c|} 
Jp to $70 \%$ response rate \\
PFS $10 \mathrm{mo} /$ nd. RND
\end{tabular} & \begin{tabular}{|c|} 
NCT00477386 \\
(conflicting results to \\
doi: \\
10.1038/bjc. 2014.116 \\
with recurrent cancer \\
patients) \\
\end{tabular} \\
\hline & Relapsed or refractory & 80 & $\begin{array}{c}\text { Belinostat }+ \\
\text { Earboplatin + paclitaxe }\end{array}$ & $\begin{array}{c}\text { 43\% clinical benefit/nd. } \\
\text { RND }\end{array}$ & NCT00421889 \\
\hline
\end{tabular}




\begin{tabular}{|c|c|c|c|c|c|}
\hline & Stage III/IV & 18 & $\begin{array}{c}\text { Vorinostat + paclitaxel } \\
+ \text { carboplatin }\end{array}$ & \begin{tabular}{|c|}
$18 / 18(100 \%)$ PFS up to \\
24 months/nd. RND
\end{tabular} & NCT00976183 \\
\hline \multirow{2}{*}{ Cervix } & Stage IVB & 143 & $\begin{array}{l}\text { VPA + hydralazine + } \\
\text { cisplatin + topotecan }\end{array}$ & $\begin{array}{l}\text { Survival benefit/Phase } \\
\text { III randomized trial }\end{array}$ & NCT00532818 \\
\hline & Relapsed and metastatic & 25 & Decitabine + cisplatin & $\begin{array}{c}-62 \% \text { response rate } / \mathrm{nd} . \\
\text { RND }\end{array}$ & [14] \\
\hline \multirow{3}{*}{ Breast } & $\mathrm{ER}+$ / exemestane-resistant & 600 & $\begin{array}{l}\text { Entinostat + } \\
\text { exemestane }\end{array}$ & $\begin{array}{c}\text { Survival benefit/Phase } \\
\text { II/randomized trial }\end{array}$ & NCT02115282 \\
\hline & Male/high stage & 54 & $\begin{array}{c}\text { Vorinostat + paclitaxel } \\
+ \text { bevacizumab }\end{array}$ & \begin{tabular}{|}
$49 \%$ stable disease $/ \mathrm{nd}$ \\
RND
\end{tabular} & NCT00368875 \\
\hline & Hormone-therapy resistant & 43 & Vorinostat + tamoxifen & $\begin{array}{l}6 \text { mo stable dis. } \\
\sim 40 \% / \text { nd. RND } \\
\end{array}$ & NCT00365599 \\
\hline \multirow{3}{*}{ NSCLC } & $\begin{array}{c}\text { Progressive } \& \text { metastatic } \\
\text { with hypermethylation of }> \\
2 / 4 \text { genes* }\end{array}$ & 10 & $\begin{array}{l}\text { Azacitidine }+ \\
\text { entinostat }\end{array}$ & $\begin{array}{c}\text { Remission occurred/nd. } \\
\text { RND }\end{array}$ & NCT01935947 \\
\hline & $\begin{array}{c}\text { Stage IIIB/IV with E- } \\
\text { cadherin (+) }\end{array}$ & 70 & Erlotinib + entinostat & $\begin{array}{l}\text { Randomized phase II } \\
\text { trial/survival benefit }\end{array}$ & NCT00750698 \\
\hline & Stage IIIB/IV & 62 & $\begin{array}{c}\text { Vorinostat/placebo + } \\
\text { (carboplatin+ } \\
\text { paclitaxel) }\end{array}$ & $\begin{array}{l}34 \% \text { vs. } 12.5 \text { response; } \\
\text { NSS survival } \\
\text { benefit/Phase II } \\
\text { randomized trial }\end{array}$ & $\begin{array}{c}\text { NCT00481078 } \\
\text { conflicting results } \\
\text { with trials } \\
\text { [NCT01413750 \& } \\
\text { NCT00473889) }\end{array}$ \\
\hline \multirow{4}{*}{$\begin{array}{l}\text { Multiple } \\
\text { myeloma }\end{array}$} & $\begin{array}{l}\text { Progressive/non- } \\
\text { refractory }\end{array}$ & 317 & $\begin{array}{c}\text { Bortezomib + } \\
\text { vorinostat }\end{array}$ & \begin{tabular}{|c|} 
Randomized phase III \\
trial; significant \\
survival benefit ( $7.6 \mathrm{vs}$. \\
$6.8 \mathrm{mo}$ ) \\
\end{tabular} & NCT00773747 \\
\hline & Relapsed & 768 & $\begin{array}{c}\text { Panabinostat/placebo } \\
+ \text { Dexamethasone }+ \\
\text { Bortezomib }\end{array}$ & $\begin{array}{c}\text { Phase III randomized } \\
\text { trial: ORR: 61\% vs. } \\
\text { 55\%; NSS } \\
\end{array}$ & NCT01023308 \\
\hline & $\begin{array}{l}\text { Relapsed \&Bortezomib } \\
\text { refractory }\end{array}$ & 55 & $\begin{array}{c}\text { Panabinostat }+ \\
\text { Dexamethasone }+ \\
\text { Bortezomib } \\
\end{array}$ & $\begin{array}{c}\sim 52 \% \text { response; } \sim 34 \% \\
\text { ORR/nd. RND }\end{array}$ & NCT01083602 \\
\hline & Relapsed or refractory & 40 & $\begin{array}{c}\text { Romidepsin }+ \\
\text { bortezomib }+ \\
\text { dexamethasone }\end{array}$ & \begin{tabular}{|c|}
$25 \%$ response rate \\
(durable response)/nd. \\
RND
\end{tabular} & NCT00431990 \\
\hline $\begin{array}{c}\text { Hepato- } \\
\text { cellular } \\
\text { carcinoma }\end{array}$ & $\begin{array}{l}\text { Progressive \& advanced } \\
\text { \&sorafenib refractory }\end{array}$ & 57 & $\begin{array}{l}\text { Resminostat +/- } \\
\quad \text { sorafenib }\end{array}$ & $\begin{array}{c}\text { Survival } \\
\text { benefit/comparative } \\
\text { phase I/II trial/ nd. RND }\end{array}$ & NCT00943449 \\
\hline \begin{tabular}{|c|}
$\begin{array}{c}\text { Polycythemia } \\
\text { vera }\end{array}$ \\
\end{tabular} & Unresponsive to TMD of $\mathrm{HC}$ & 44 & Givinostat + TMD of HC & $\begin{array}{c}\text { Clinical response/nd. } \\
\text { RND }\end{array}$ & $\begin{array}{c}\text { DOI: } \\
\text { 10.1111/bjh.12332 }\end{array}$ \\
\hline Colorectal & Irinotecan-resistant & 108 & $\begin{array}{l}\text { Guadecitabine + } \\
\text { irinotecan }\end{array}$ & $\begin{array}{c}-60 \% \text { response rate/nd. } \\
\text { RND }\end{array}$ & NCT01896856 \\
\hline $\begin{array}{l}\text { Kidney } \\
\text { cancer }\end{array}$ & Unresectable or metastatid & 37 & $\begin{array}{l}\text { Vorinostat + } \\
\text { Bevacizumab }\end{array}$ & $\begin{array}{c}\text { 69\% response rate/nd. } \\
\text { RND }\end{array}$ & NCT00324870 \\
\hline
\end{tabular}

*interm: intermediate; AML: acute myeloid leukemia; CMML: chronic myelomonocyticleukaemia; NHL: non-Hodgkin lymphoma; HL: Hodgkin's lymphoma; PTCL: Peripheral T cell lymphoma; SCT: Stem cell transplant; NSCLC: Non-small cell lung cancer; 4 genes include: APC, RASSF1A, CDH13, and CDKN2A; nd. RND: Needs randomized trials for confirmation

Table 1: Selected studies representing most efficient evidence on epigenetic therapies in high-risk cancer populations

\section{References}

1. Jones PA, Issa JP, Baylin S (2016) Targeting the cancer epigenome for therapy. Nat Rev Genet 17(10): 630-641.
2. Sato T, Issa JJ, Kropf P (2017) DNA Hypomethylating Drugs in Cancer Therapy. Cold Spring Harb Perspect Med 7(5) pii: a026948.

3. Yun S, Vincelette ND, Abraham I, Robertson KD, Fernandez Zapico ME, et al. (2016) Targeting 
epigenetic pathways in acute myeloid leukemia and myelodysplastic syndrome: a systematic review of hypomethylating agents trials. Clin Epigenetics 8: 68.

4. Borthakur G, Ahdab SE, Ravandi F, Faderl S, Ferrajoli A, et al. (2008) Activity of decitabine in patients with myelodysplastic syndrome previously treated with azacitidine. Leuk Lymphoma 49(4): 690-695.

5. Mottamal M, Zheng S, Huang TL, Wang G (2015) Histone deacetylase inhibitors in clinical studies as templates for new anticancer agents. Molecules 20(3): 3898-3941.

6. Galanis E, Jaeckle KA, Maurer MJ, Reid JM, Ames $\mathrm{MM}$, et al. (2009) Phase II trial of vorinostat in recurrent glioblastomamultiforme: a north central cancer treatment group study. J Clin Oncol 27(12): 2052-2058.

7. Linnekamp JF, Butter R, Spijker R, Medema JP, van Laarhoven HW (2017) Clinical and biological effects of demethylating agents on solid tumours - A systematic review. Cancer Treat Rev 54: 10-23.

8. Ronnekleiv Kelly SM, Sharma A, Ahuja N (2017) Epigenetic therapy and chemosensitization in solid malignancy. Cancer Treat Rev 55: 200-208.

9. Craddock CF, Houlton AE, Quek LS, Ferguson P, Gbandi E, et al. (2017) Outcome of Azacitidine
Therapy in Acute Myeloid Leukemia Is not Improved by Concurrent Vorinostat Therapy but Is Predicted by a Diagnostic Molecular Signature. Clin Cancer Res 23(21): 6430-6440

10. Prebet T, Sun Z, Figueroa ME, Ketterling R, Melnick $A$, et al. (2014) Prolonged administration of azacitidine with or without entinostat for myelodysplastic syndrome and acute myeloid leukemia with myelodysplasia-related changes: results of the US Leukemia Intergroup trial E1905. J Clin Oncol 32(12): 1242-1248.

11. Taheri S (2017) In vitro Evidence on Associations between MicroRNAs and Response to Therapy in Gastric Cancer: Report from the Encyclopedia Amlashica Systematic Reviews. Madridge J Can Stu Res 1(1): 12-33.

12. Schuh AC, Dohner H, Pleyer L, Seymour JF, Fenaux $\mathrm{P}$, et al. (2017) Azacitidine in adult patients with acute myeloid leukemia. Crit Rev Oncol Hematol 116: 159-177.

13. Treppendahl MB, Kristensen LS, Gronbæk K (2014) Predicting response to epigenetic therapy. J Clin Invest 124(1): 47-55.

14. Pohlmann P, DiLeone LP, Cancella AI, Caldas AP, Dal Lago L, et al. (2002) Phase II trial of cisplatin plus decitabine,a new DNA hypomethylating agent, in patients with advanced squamous cellcarcinoma of the cervix. Am J ClinOncol 25(5): 496-501. 